\title{
High-order-harmonic generation from dense water microdroplets
}

\author{
Heiko G. Kurz, ${ }^{1,2,{ }^{*}}$ Daniel S. Steingrube, ${ }^{1,2}$ Detlev Ristau, ${ }^{2,3}$ Manfred Lein, ${ }^{2,4}$ Uwe Morgner, ${ }^{1,2,3}$ and Milutin Kovačev ${ }^{1,2}$ \\ ${ }^{1}$ Leibniz Universität Hannover, Institut für Quantenoptik, Welfengarten 1, D-30167 Hannover, Germany \\ ${ }^{2}$ QUEST, Centre for Quantum Engineering and Space-Time Research, Welfengarten 1, D-30167 Hannover, Germany \\ ${ }^{3}$ Laser Zentrum Hannover e.V., Hollerithallee 8, D-30419 Hannover, Germany \\ ${ }^{4}$ Leibniz Universität Hannover, Institut für theoretische Physik, Appelstrasse 2, D-30167 Hannover, Germany
}

(Received 10 July 2012; published 7 June 2013)

\begin{abstract}
We report on high-order-harmonic generation from micrometer-sized liquid water droplets. In pump-probe experiments, the influence of the time delay onto the emission of harmonic radiation is systematically studied. Phase-matching aspects are observed by controlling the focal position and the intensity of the probe pulse. The spatiotemporal dynamics of the droplet during interaction with intense laser pulses are studied by controlling the intensity of the pump pulse. We find transient phase-matching conditions and the expansion dynamics of the droplet to be of major influence on the harmonic yield.
\end{abstract}

DOI: 10.1103/PhysRevA.87.063811

PACS number(s): 42.65.Ky, 42.65.Re, 42.50.Nn

\section{INTRODUCTION}

High-order-harmonic generation (HHG) from atoms and molecules is an established source of coherent extremeultraviolet (XUV) radiation [1-3]. The unique characteristics of the radiation make it possible to perform experiments which require a high temporal and a high spatial resolution, revealing phenomena on the attosecond time scale [4-6]. Moreover, harmonic radiation exhibits high spatial $[7,8]$ and temporal coherence properties $[9,10]$. Today, harmonic radiation is generated with pulse energies in the $\mu J$ range [11-13]. In high-order-harmonic spectroscopy, pump-probe experiments are performed to study molecular dynamics during chemical reactions [14] or rotational dynamics of molecules and their influence onto the emission of XUV radiation [15-18]. When harmonic radiation is generated in a molecular target, the "single-active-electron approximation" fails to explain the observations and multielectron effects have to be taken into account [19]. Structural information can be extracted from the harmonic signal [16] with its climax in tomographic imaging of molecular orbitals $[18,20,21]$. Recently, attention turned toward HHG in water molecules [22-26], due to the fact that it is of nontrivial structure. Therein, the contributions of different molecular orbitals to the harmonic signal could be identified [27].

Apart from the above described single-emitter response in atoms or molecules, harmonic radiation is generated in an ensemble of emitters under experimental conditions. That is why the coherent sum of the emitted XUV photons within the target forms the net harmonic signal. Therefore, not only microscopic but also macroscopic effects have to be taken into account. The phases of all the emitters have to be matched, in order to maximize the harmonic yield. Different terms such as geometric, dispersive, and atomic phase contributions have to be accounted for and can be tuned by experimental parameters, such as the target geometry, the target density or the focal position.

*Corresponding author: kurz@iqo.uni-hannover.de
Experimentally, HHG has been performed using atoms [1] and molecules [28] as a target in several thermodynamical states, such as gaseous, liquid, or solid state [1,22,23,29-33]. Moreover, different geometries with impact on the phasematching conditions have been used for HHG [22,23,34-36]. Most of the phase-matching studies have been performed on gaseous, atomic targets. Therein, mainly three different geometries have been applied: the gas jet [34], the finite gas cell [35,37], and the semi-infinite gas cell [36]. In contrast to the gaseous state, HHG from a liquid target is rather unexplored. Only few measurements have been performed concerning liquid targets. DiChiara et al. [23] observed harmonic radiation in a liquid jet consisting of water and heavy water, using a midinfrared laser source. Harmonic radiation up to the thirteenth order was observed with different yield for both isotopes. In the work of Flettner et al. [22], a liquid-water droplet setup for HHG was used, where a prepulse expands the water droplet before a second pulse generates high-order-harmonic radiation. In these pump-probe experiments, a transition from incoherent to coherent radiation with increasing pump-probe time delay was observed and harmonic radiation up to the twenty-seventh order was generated. While Flettner et al. demonstrated a proof-of-principle experiment, further research has not been performed on liquid droplets as source for HHG. Moreover, little theoretical research has been done so far [38].

Although the liquid droplet as source for HHG is not well understood, its unique characteristics feature some remarkable benefits, when compared to either target, the gaseous, or the solid state. Due to its geometry, the droplet is a masslimited target and therefore the number of particles participating in the nonlinear interaction process can be estimated. Moreover, debris-free HHG becomes possible along with a high-density target. In pump-probe experiments transitions between different thermodynamical states can be accessed. The versatility of the source becomes obvious when the large range of different target materials is considered, as many liquids become accessible for HHG. In this paper, we present detailed systematical investigations of phasematching conditions and pump-probe experiments during the interaction of an intense laser field with liquid-water droplets. 


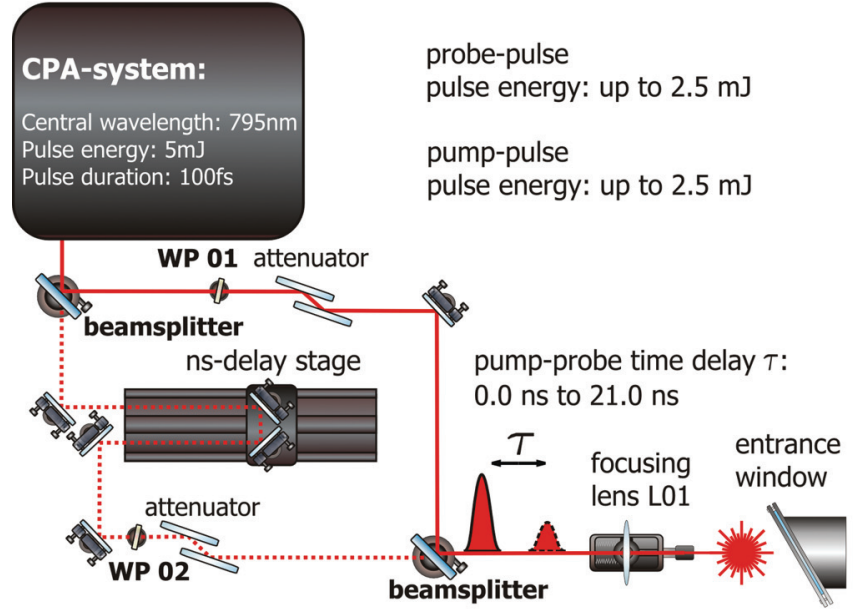

(a)

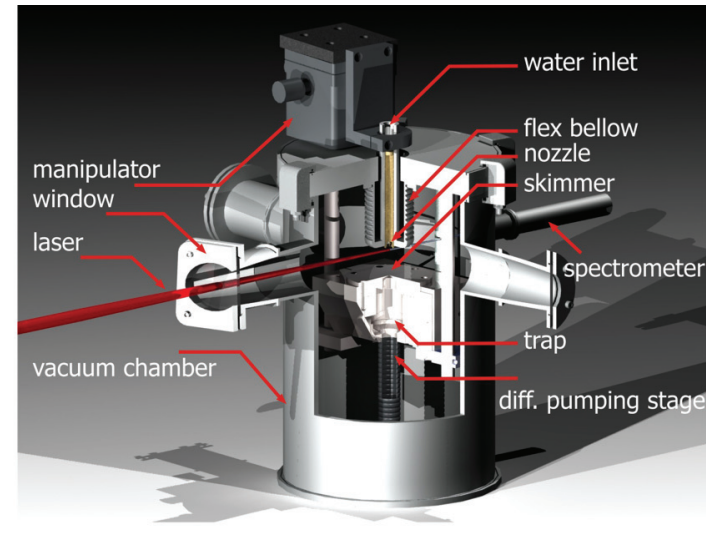

(b)

FIG. 1. (Color online) (a) Optical setup for the generation of pump and probe pulse by a Mach-Zehnder interferometer. The pump-probe time delay $\tau$ is adjusted by a linear positioning stage. (b) Setup of the liquid droplet source. See text for description.

\section{EXPERIMENTAL SETUP}

As a laser source for HHG from water droplets, a chirpedpulse-amplification system (improved Alpha 10C/CS, Thales) is applied, delivering $100-\mathrm{fs}$ pulses centered at $792 \mathrm{~nm}$. A pulse energy of up to $5 \mathrm{~mJ}$ at a repetition rate of $20 \mathrm{~Hz}$ has been used for performing the experiments. The pulse duration has been measured with a home-built second-order single-shot autocorrelator. For the pump-probe experiments, a Mach-Zehnder interferometer has been set up [see Fig. 1(a)]. In every arm, there is an attenuator to vary the pump-probe energy ratio. Each attenuator consists of a half-wave plate (WP 1 and WP 2) and two thin film polarizers reflecting the $s$-polarized part of the beam. Within the interferometer arm serving the pump pulse, a manual translation stage is placed adjusting the pump-probe time delay $\tau$. The pulses are focused non collinearly into the vacuum chamber of the droplet source with a $\mathrm{CaF}_{2}$ lens (L01, $f=500 \mathrm{~mm}, z_{R}=5.42 \mathrm{~mm}$ ), passing a $\mathrm{CaF}_{2}$ entrance window of $1 \mathrm{~mm}$ thickness.

The high-harmonic chamber consists of a capillary nozzle (Micro Jet Components) with $10 \mu \mathrm{m}$ diameter and a trap for the liquid droplets [see Fig. 1(b)]. Using a nozzle with piezoelectric aperture, a hydrodynamic instability is imprinted onto the water jet. Thus, a determined series of micrometersized water droplets is created in a certain distance from the nozzle $[39,40]$. Their diameter under experimental conditions is determined to be $15 \mu \mathrm{m}$. The nozzle is fed by a capillary and a HPLC pump (Knaur,WellChrom K-120), supplying a background pressure of $55 \mathrm{~atm}$ of deionized water. After a few $\mathrm{cm}$ of propagation in the vacuum, the droplets are being shot through a heated skimmer into a differential pumping stage. Although the time the droplets spend in the vacuum is very short, some molecules evaporate from its surface. In order to prevent a contamination of the vacuum, a liquid $\mathrm{N}_{2}$ trap has been installed, freezing the water molecules. Under experimental conditions, a pressure of $3.0 \times 10^{-5}$ mbar has been achieved within the high-harmonic chamber, which is sufficiently low for suppressing reabsorption effects.

Another differential-pumping stage has been installed along the propagation direction of the harmonic radiation. The
XUV radiation is spectrally resolved by a grazing incidence spectrometer (LHT 30, Horiba-Jobin-Yvon, 500 lines $/ \mathrm{mm}$ ) with an attached microchannel plate detector.

\section{RESULTS AND DISCUSSION}

The harmonic intensity is measured in dependence of the pump-probe time delay, the focal position, and of the intensity of either the pump and the probe pulse.

(a) Harmonic spectra. Figure 2(a) shows a spectrum of the harmonic radiation at a pump-probe time delay of $3.5 \mathrm{~ns}$. The spectrum was recorded at an intensity of the probe pulse of $3.7 \times 10^{14} \mathrm{~W} / \mathrm{cm}^{2}$. The graph shows the typical features of harmonic spectra known from gaseous targets. It can be divided into three regions. This includes a decay of the harmonic intensity according to perturbation theory in the orders nine and eleven, followed by the plateau region in the orders 13 to 19 and finally, the cutoff region, ending with the twenty-seventh harmonic order. This is below the calculated cutoff from $E_{\text {cutoff }}=I_{p}+3.17 U_{p}[2,41]$, where $I_{p}$ denotes the ionization potential of water $(12.6 \mathrm{eV})$ and $U_{p}$ denotes the ponderomotive potential. The twenty-seventh harmonic order is the highest observed order during all of our experiments.

(b) Calculations on droplet expansion. When the droplet is hit by the pump pulse, it starts expanding and thus performs a transition from the liquid state to a gaseous state, and depending on the intensity of the pump pulse even a plasma can be ignited. Thus different thermodynamical states can be accessed by the probe pulse to generate harmonic radiation. Two parameters critically influence the spatiotemporal dynamics of the target: the intensity of the pump pulse and the time delay between pump and probe pulse. In order to estimate the density of the target at a given time after being hit by the pump pulse, calculations on the density variation of the droplet have been performed. Therefore, a model developed by Puell et al. [42] has been used, treating the interaction of a solid-state target with intense laser pulses. This approach has been validated for the liquid droplet [43]. From the model, an intensity-dependent expansion velocity can be derived, which 


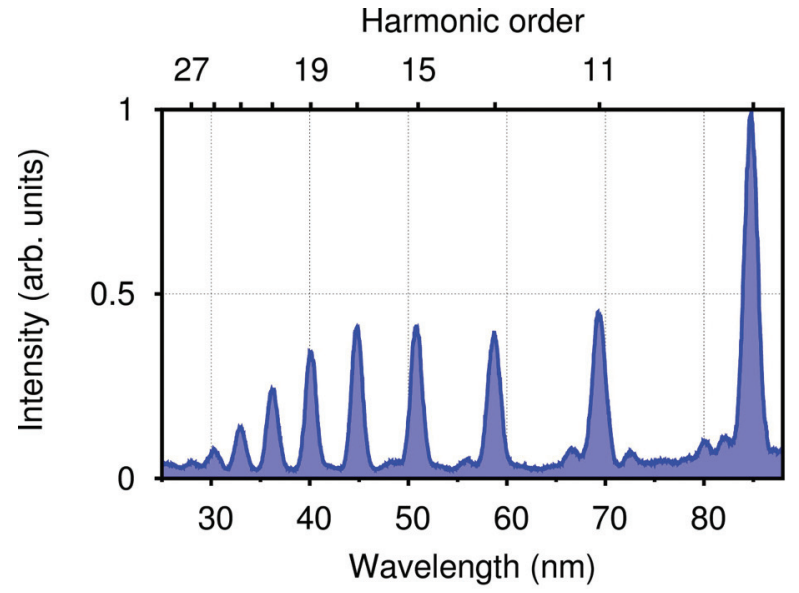

(a)

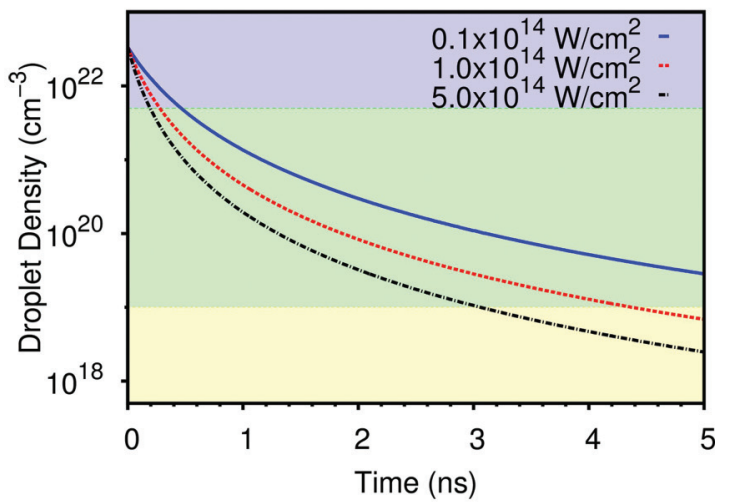

(b)

FIG. 2. (Color online) (a) Harmonic intensity versus wavelength and harmonic order. The spectrum was taken at a pump-probe time delay of $\tau=3.5 \mathrm{~ns}$ at an intensity of the pump pulse of $7.0 \times 10^{14} \mathrm{~W} / \mathrm{cm}^{2}$, an intensity of the probe pulse of $3.7 \times 10^{14} \mathrm{~W} / \mathrm{cm}^{2}$ and a focal parameter of $z<0 \mathrm{~mm}$. (b) Calculated density of the target as function of time for three intensities of the pump pulse. The colored areas roughly mark different thermodynamical densities. Blue: liquid and clustered media, green: clustered media and high-density gas, yellow: gas.

scales as

$$
v_{\text {exp }} \propto I^{2 / 9} .
$$

Assuming a uniform velocity and starting with a density of the droplet of $d_{\text {drop }}=3.35 \times 10^{22} \mathrm{~cm}^{-3}$, the intensity-dependent density as function of time can be calculated and scales as

$$
d_{\text {drop }} \propto I^{-2 / 3}
$$

with intensity. Within the calculations, a degree of ionization of $Z=1$ is assumed. The model reproduces accurately the expansion velocities, measured in a water jet during interaction with a laser pulse [44].

Figure 2(b) depicts the decrease of the density of the target with time for three different intensities of the pump pulse. Considering a fixed intensity of $1 \times 10^{14} \mathrm{~W} / \mathrm{cm}^{2}$ (red line), the density develops as followed: For times $\tau<0.3 \mathrm{~ns}$ the target has liquid-water density, which is around $d_{\mathrm{drop}} \approx 10^{22} \mathrm{~cm}^{-3}$. With increasing time, the target further expands and performs a transition from liquid to a dense gas. This high-density target is given for delays $\tau<4.0 \mathrm{~ns}$ with densities between $10^{22} \mathrm{~cm}^{-3}$ and $10^{19} \mathrm{~cm}^{-3}$. Further increase of time leads to a lowering of the density comparable to dry gases with $d_{\text {drop }} \leqslant 10^{19} \mathrm{~cm}^{-3}$. Increasing the intensity of the pump pulse leads to a more rapid decrease of the density.

(c) Influence of the pump-probe time delay. To gain more insight into the spatiotemporal behavior of the target, we exploit our pump-probe setup to vary the delay between the pump and the probe pulse. Figure 3(a) shows the harmonic intensity as a function of the pump-probe time delay and the harmonic order. With increasing delay, a raise in harmonic intensity and an increase in harmonic cutoff can be observed. For a more detailed analysis of the increase of the harmonic cutoff as function of the pump-probe time delay, Fig. 3(b) shows normalized spectra from the data presented in Fig. 3(a) at four time delays $\tau$. The highest observed harmonic order starts at the fifteenth harmonic order at $\tau=0.4 \mathrm{~ns}$ and increases to the twenty-fifth harmonic order at a time delay of $3.0 \mathrm{~ns}$.
Efficient HHG from liquid droplets depends critically on two major effects: Phase matching and unperturbed excursion of the electrons in the continuum [38]. When a droplet is hit by the pump pulse, it starts expanding and performs a transition from the liquid state to a gaseous state [see Fig. 2(b)]. Thus, a high-density target is given at small delays $\tau$ and therefore contains a potentially high amount of emitters for HHG. However, perturbation of the tunneled electrons by other particles becomes more probable during excursion in the continuum. As a consequence, coherence is lost and the harmonic yield is suppressed for short delays [see Fig. 3(a)]. At larger delays $\tau$, the droplet is more expanded, which leads to less distortion of the electron trajectories and reduced absorption of the generated radiation due to a lower density [see Fig. 2(b)]. Thus, increasing the delay leads to a rise in harmonic yield and extends the cutoff of the harmonic radiation due to unperturbed electronic motion, which is in agreement with the results from Flettner et al. But in contrast to the work of Flettner et al. [22], we were able to observe harmonic radiation at small delay times throughout our measurements (down to $0.2 \mathrm{~ns}$ ), and only a negligible amount of incoherent radiation has been detected. Though being in a comparable intensity regime as in Ref. [22], no transition from incoherent emission to coherent emission of radiation has been detected. The discrepancy is probably due to different experimental setup [45]. In particular, two aspects can provide an explanation of our result: large distances between the droplet and the detector, in our case $1.4 \mathrm{~m}$, and narrow entrance slits of the spectrometer. Due to a long distance between droplet and detector, less incoherent radiation reaches the detector because of its divergent nature of emission. In contrast, harmonic radiation is emitted directed and more easily reaches the detector. In combination with narrow entrance and exit slits of the spectrometer, even less incoherent radiation can be observed. Therefore, the presence of a transition from incoherent contributions in the measured spectrum strongly depends on the details of the setup. 


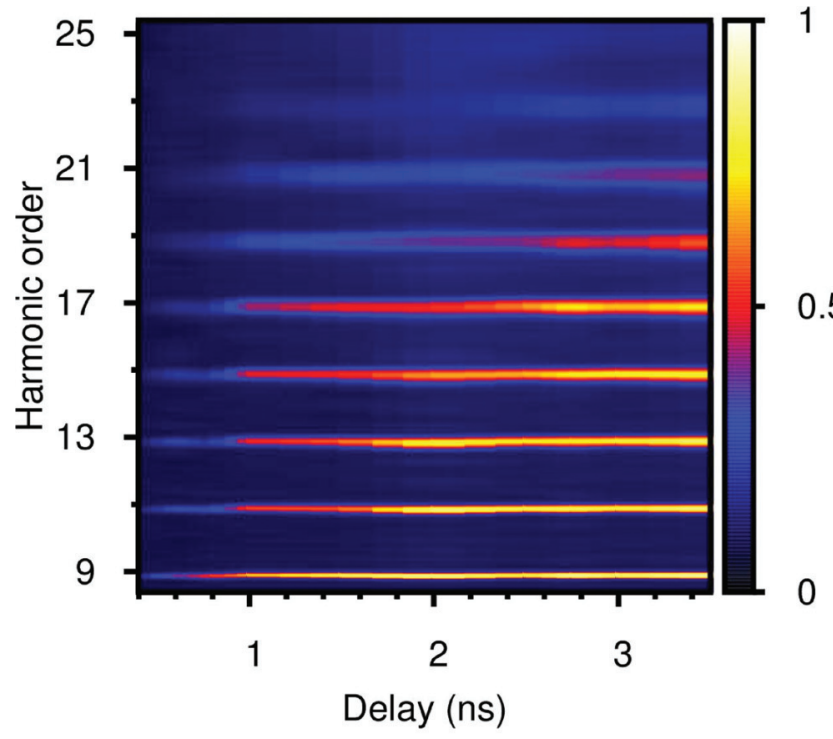

(a)

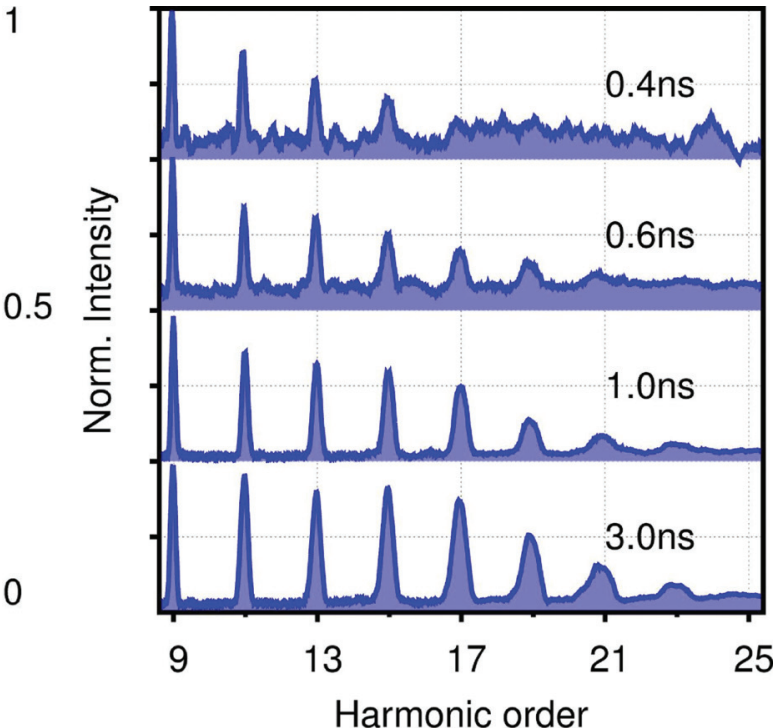

(b)

FIG. 3. (Color online) (a) Harmonic intensity versus order and pump-probe time delay. The spectra were taken at an intensity of the pump pulse and of the probe pulse of $4.5 \times 10^{14} \mathrm{~W} / \mathrm{cm}^{2}$. The focal position was $z=-2 \mathrm{~mm}$. (b) Harmonic intensity versus order at four different pump-probe time delays. The spectra show normalized data from Fig. 3(a). Note that the broadening of the harmonic lines with increasing order originates from its presentation as a function of harmonic order and is chosen for better visibility.

In Fig. 4, spectra of harmonic radiation at three different pump-probe delays $\tau$ are shown, concentrating on large time delays. For the reasons given above, the harmonic yield increases between $1.5 \mathrm{~ns}$ and $12.5 \mathrm{~ns}$ where it reaches a maximum. Between $12.5 \mathrm{~ns}$ and $21.0 \mathrm{~ns}$, however, a decrease of the harmonic yield is observed. We interpret this behavior as follows: At delay times $\tau>12.5 \mathrm{~ns}$, the target has expanded so far that part of it has propagated outside the focal region. Along with the expansion of the target, its density decreases to the order of $10^{16} \mathrm{~cm}^{-3}$. Thus, less particles contribute to the harmonic signal. Altogether, we demonstrate the probing

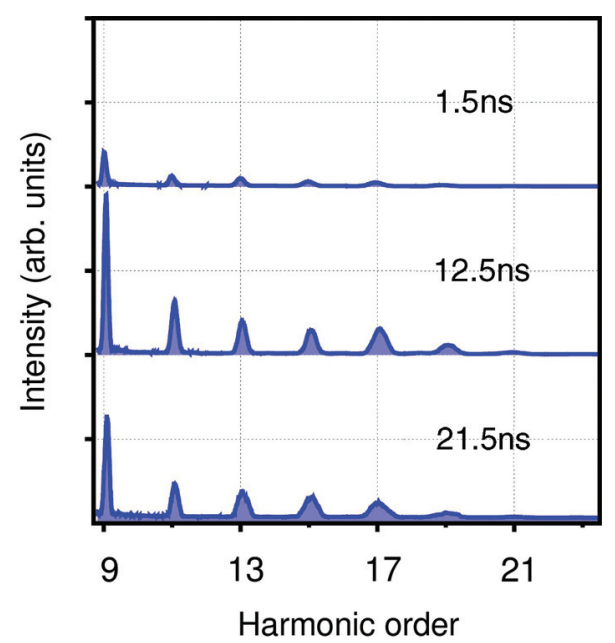

FIG. 4. (Color online) Harmonic intensity versus order at three different pump-probe time delays. All parameters were set comparable to Fig. 3. For better visibility, the data is plotted versus harmonic order. of different density states of the target by variation of the pump-probe time delay.

(d) Influence of the focal position. In order to prove our calculations in Fig. 2 for densities in the order of $10^{20} \mathrm{~cm}^{-3}$, phase-matching aspects were studied by variation of the focal position. Figure 5(a) depicts the harmonic intensity as a function of the harmonic order and the focal position $z$ at a pump-probe time delay of $1.0 \mathrm{~ns}$. For positions $z<0 \mathrm{~mm}$, the focus is placed before the droplet with respect to the propagation direction. A maximum in the harmonic yield can be observed for $z=-2 \mathrm{~mm}$. The harmonic intensity drops when the focus is placed directly on the droplet $(z \approx 0 \mathrm{~mm})$. A second maximum in harmonic intensity can be seen at $z=1 \mathrm{~mm}$, i.e., when the focus is positioned behind the droplet. To show this behavior in more detail, outlines of the eleventh and nineteenth harmonic order from Fig. 5(a) are given in Figs. 5(b) and 5(c), respectively. The maxima in harmonic intensity at $z=-2 \mathrm{~mm}$ and $z=1 \mathrm{~mm}$ and of the minimum at $z \approx 0 \mathrm{~mm}$ are clearly observed.

We interpret our observations in analogy to the phasematching conditions in a gas jet, described by Salières et al. $[34,46]$. The maximum at $z=-2.0 \mathrm{~mm}$ is referred to optimum phase-matching conditions on the axis. This means the variation of the geometric phase and the radially induced atomic phase compensate each other, resulting in low phase variation on the axis. This corresponds to phasematching conditions where the short trajectory is preferred. The maximum at $z=1.0 \mathrm{~mm}$ can be explained by good phase-matching conditions at an off-axis distance, which means the variation of the total phase is low, resulting in proper phase-matching conditions for the long trajectory. Our measurements are qualitatively in good agreement with the phase-matching conditions observed in a gas jet, but note that the amplitude of the maxima is inverted $[34,46]$. Moreover, 


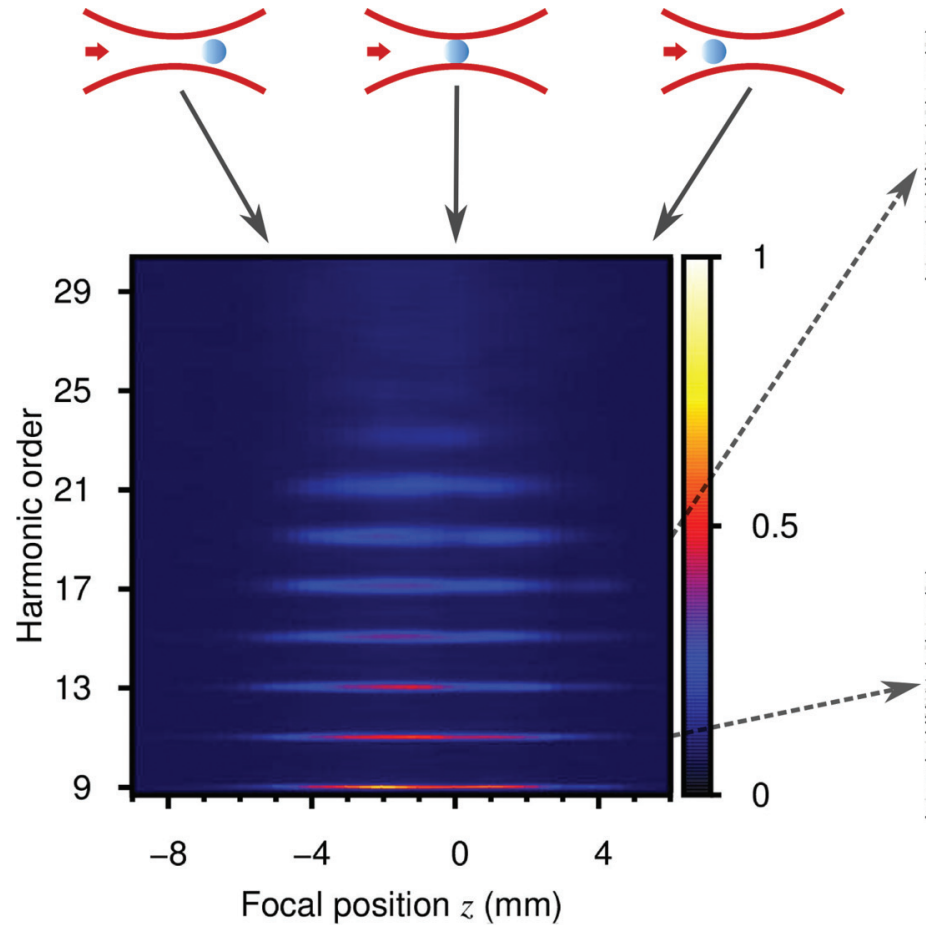

(a)

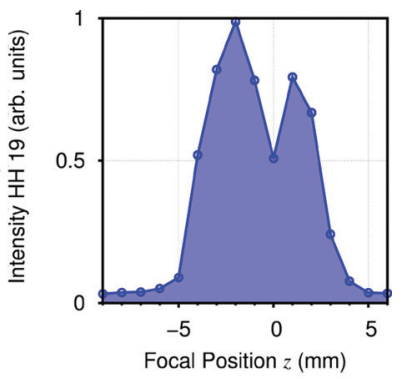

(b)

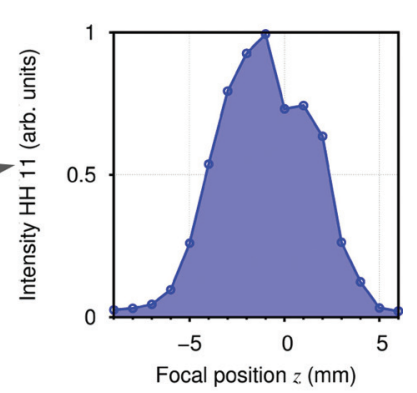

(c)

FIG. 5. (Color online) (a) Harmonic intensity versus order and focal position at fixed delay $\tau=1.0 \mathrm{~ns}$. The intensity of the pump pulse and of the probe pulse were set to $4.5 \times 10^{14} \mathrm{~W} / \mathrm{cm}^{2}$. The schematics at the top mark the different positions of the focus relative to the droplet, with respect to the propagation direction shown as red arrow. (b) Outline of the nineteenth harmonic order and (c) the eleventh harmonic order along the focal position $z$.

the results agree with our calculations of the density of the target, which is a dense gas with $d_{\text {drop }}=2.1 \times 10^{20} \mathrm{~cm}^{-3}$ [cf. Fig. 2(b)].

(e) Influence of the pump-probe intensity. Figure 6(a) depicts the harmonic yield versus order and intensity of the pump pulse. The intensity of the probe pulse is kept at a constant level of $3.66 \times 10^{14} \mathrm{~W} / \mathrm{cm}^{2}$ at a pump-probe time delay of $\tau=$ $1.0 \mathrm{~ns}$. At a pump-pulse intensity below $1.0 \times 10^{14} \mathrm{~W} / \mathrm{cm}^{2}$ only the lower harmonic orders are generated, though with a poor signal to noise ratio. Increasing the intensity of the pump pulse, an increase of the harmonic yield becomes observable. A maximum in harmonic intensity is reached around an intensity of $3.3 \times 10^{14} \mathrm{~W} / \mathrm{cm}^{2}$. Additionally, the highest observed harmonic order extends up to the twenty-fifth order. For a more detailed observation of this behavior, an outline of the twenty-fifth harmonic order is given in Fig. 7. The twenty-fifth harmonic order appears at an intensity of $2.0 \times 10^{14} \mathrm{~W} / \mathrm{cm}^{2}$ and reaches its maximum yield around $3.0 \times 10^{14} \mathrm{~W} / \mathrm{cm}^{2}$. Further increase of the pump-pulse intensity to more than $3.3 \times 10^{14} \mathrm{~W} / \mathrm{cm}^{2}$ results in a decrease of the harmonic cutoff and an increase of incoherent radiation.

From the measurements, two effects can be distinguished that influence the harmonic yield and the harmonic cutoff and are sensitive to the pump-pulse intensity. The first one is the intensity-dependent expansion of the droplet. The second effect is the rising density of free electrons due to ionization. As stated before [see Eq. (2)], the density of the expanding droplet can be calculated from its expansion velocity, which scales with intensity [42]. Thus, an increase in intensity of the pump pulse leads to an accelerated expansion and therefore a reduced density of the target at the time of the probe pulse. From our calculations, we estimate the density of the droplet at an intensity of $0.6 \times 10^{14} \mathrm{~W} / \mathrm{cm}^{2}$ to be $5.9 \times 10^{20} \mathrm{~cm}^{-3}$. The density lowers to $2.2 \times 10^{20} \mathrm{~cm}^{-3}$ at an intensity of $4.0 \times 10^{14} \mathrm{~W} / \mathrm{cm}^{2}$. This means the density of the target is reduced by nearly a factor of three along the $x$ axis of Fig. 6(a). As a result, the low harmonic yield at intensities of the pump pulse below $2.0 \times 10^{14} \mathrm{~W} / \mathrm{cm}^{2}$ can be attributed to the high density of the target at which the distortion of the electronic trajectories is present. Moreover, absorption effects efficiently suppress harmonic generation at high density. At moderate intensities of the pump pulse $\left(2.0 \times 10^{14} \mathrm{~W} / \mathrm{cm}^{2}<I<3.3 \times\right.$ $10^{14} \mathrm{~W} / \mathrm{cm}^{2}$ ), the droplet becomes more expanded and the electron trajectories become less distorted on their excursion in the continuum together with reduced reabsorption effects. A higher harmonic yield occurs alongside with an extension of the cutoff. We interpret this extension by optimized phasematching conditions for higher-harmonic orders (see Fig. 7).

When the intensity of the pump pulse is further increased, the second effect becomes dominant, as the density of the free electrons rises dramatically. This leads to an additional contribution to the phase-matching conditions and to a defocusing effect of the fundamental beam. Thereby, a phase contribution to the mismatch between the fundamental field and the high-order harmonic radiation via a change of the refractive index is induced [47]. As a result, the cutoff is reduced and the harmonic intensity decreases. This effect becomes observable in Fig. 6(a) and especially in Fig. 7 at 


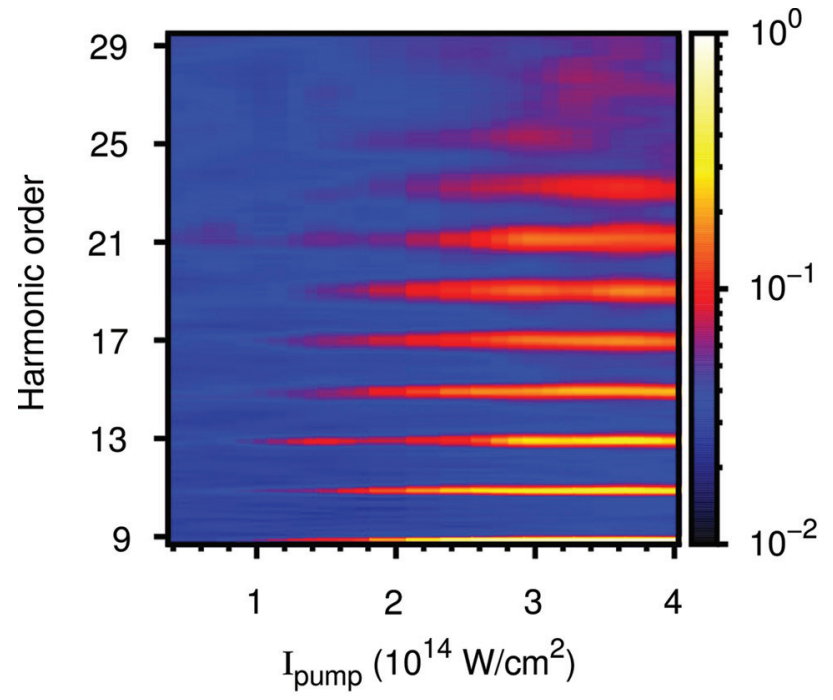

(a)

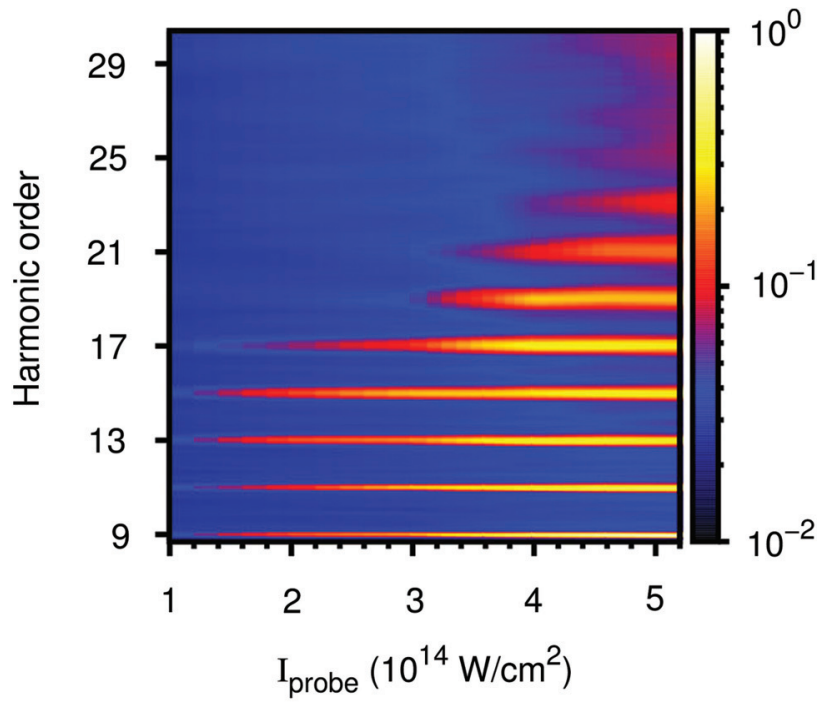

(b)

FIG. 6. (Color online) (a) Harmonic yield versus order and intensity of the pump pulse. The intensity of the probe pulse is kept at a constant level of $3.66 \times 10^{14} \mathrm{~W} / \mathrm{cm}^{2}$ at a delay of $\tau=1.0 \mathrm{~ns}$. (b) Harmonic yield versus order and intensity of the probe pulse. The intensity of the pump pulse is kept at a constant level of $2.0 \times 10^{14} \mathrm{~W} / \mathrm{cm}^{2}$ at a delay of $\tau=3.5 \mathrm{~ns}$. In both measurements, the focal position was $z=-2 \mathrm{~mm}$. Due to visibility, the data is plotted versus harmonic order.

intensities above $3.3 \times 10^{14} \mathrm{~W} / \mathrm{cm}^{2}$, where the intensity of the twenty-fifth harmonic order decreases. For the highest observed photon energies, we find an optimal intensity for the pump pulse at $I=3.0 \times 10^{14} \mathrm{~W} / \mathrm{cm}^{2}$ in the case of a delay of $\tau=1.0 \mathrm{~ns}$.

Figure 6(b) shows the intensity of the harmonic radiation versus order and intensity of the probe pulse. In this measurement, the intensity of the pump pulse is kept at a constant level of $2.0 \times 10^{14} \mathrm{~W} / \mathrm{cm}^{2}$ at a pump-probe time delay of $\tau=3.5 \mathrm{~ns}$, in order to reach the highest cutoff and to use a lower target density of $1.2 \times 10^{19} \mathrm{~cm}^{-3}$. An increase in the cutoff and harmonic signal can be seen with increasing probe intensity up to an intensity of $4.4 \times 10^{14} \mathrm{~W} / \mathrm{cm}^{2}$. Thereafter, a saturation in the harmonic signal appears and

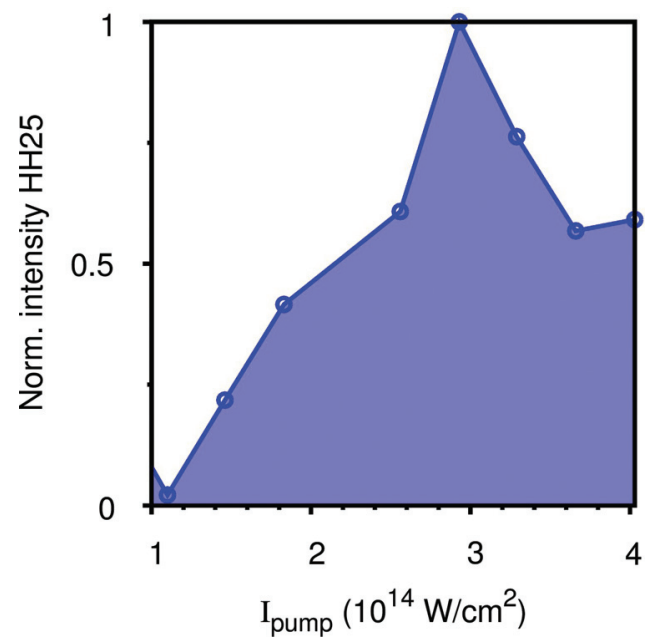

FIG. 7. (Color online) Outline from Fig. 6(a). Harmonic yield of the twenty-fifth harmonic order versus the intensity of the pump pulse. the observable harmonic order reaches up to the twentyseventh order. To determine the saturation intensity, a detailed analysis is given by outlines from Fig. 6(b) in Fig. 8 for the thirteenth [Fig. 8(a)], nineteenth [Fig. 8(b)], twenty-first [Fig. 8(c)], and the twenty-third harmonic order [Fig. 8(d)]. From these outlines, the individual saturation intensity for distinct harmonic orders can be derived. In Fig. 8(a), the saturation intensity is approximately $4.0 \times 10^{14} \mathrm{~W} / \mathrm{cm}^{2}$ but a decrease in the harmonic signal can be observed for higher intensities than the saturation intensity. Figure 8(b) features the same behavior as Fig. 8(a), but the saturation intensity has increased to approximately $4.6 \times 10^{14} \mathrm{~W} / \mathrm{cm}^{2}$. For the twenty-first harmonic order, the saturation intensity is around $5.0 \times 10^{14} \mathrm{~W} / \mathrm{cm}^{2}$ and no decrease in harmonic intensity becomes observable [see Fig. 8(c)]. For the twenty-third harmonic order, no saturation is detected [see Fig. 8(d)].

We attribute the saturation of the harmonic yield and the decrease of the harmonic signal with increasing intensity of the probe pulse to a rising density of free electrons. Increasing the intensity leads to increased ionization and an additional phase contribution, which results in a larger mismatch $[47,48]$.

(f) Spectral broadening, splitting and blueshift. In order to study the influence of a high free-electron density onto $\mathrm{HHG}$, the intensity of the pump pulse was set to $\approx 4.0 \times 10^{14} \mathrm{~W} / \mathrm{cm}^{2}$ to ignite a plasma. Figure 9(a) shows the normalized spectral profile of the thirteenth harmonic order at the pump-probe time delays $1.5 \mathrm{~ns}, 12.5 \mathrm{~ns}$, and $21.5 \mathrm{~ns}$. At short time delays $(\tau \leqslant 1.5 \mathrm{~ns})$ a shift of the harmonic order and a broadening of the spectral line can hardly be observed (full-width half maximum of the spectral line is $\Delta E=0.41 \mathrm{eV}$ ). This behavior differs, when the time delay is increased. At $\tau=12.5 \mathrm{~ns}$, the central wavelength of the harmonic order is shifted towards shorter wavelength but hardly spectrally broadened $(\Delta E=$ $0.43 \mathrm{eV})$. The effect becomes more pronounced, when the time delay is further increased to $\tau=21.5 \mathrm{~ns}$. The harmonic profile 


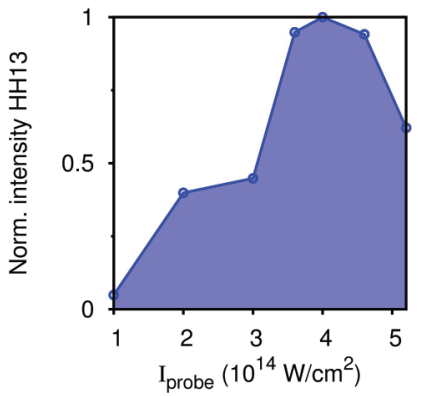

(a)

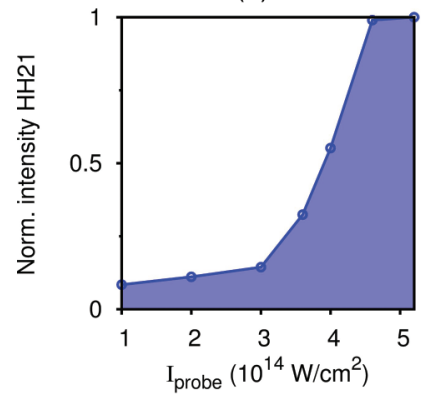

(c)

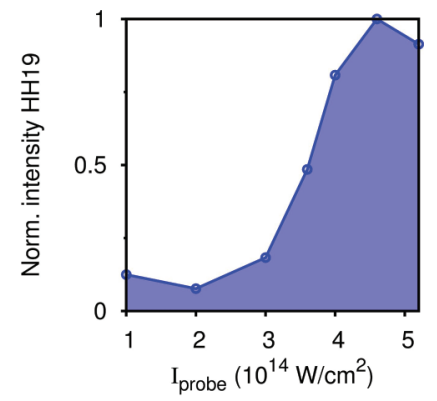

(b)

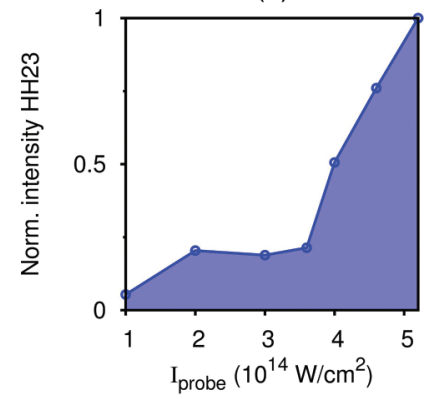

(d)

FIG. 8. (Color online) Outline from Fig. 6(b). Harmonic yield versus intensity of the probe pulse for the thirteenth (a), nineteenth (b), twenty-first (c), and twenty-third harmonic order (d).

becomes more blueshifted and is significantly broadened $(\Delta E=0.61 \mathrm{eV})$. A similar behavior can be seen for the fifteenth harmonic order [see Fig. 9(b) with $\Delta E=0.54 \mathrm{eV}$, $\Delta E=0.59 \mathrm{eV}$, and $\Delta E=0.71 \mathrm{eV}$ for $\tau=1.5 \mathrm{~ns}, 12.5 \mathrm{~ns}$, and $21.5 \mathrm{~ns}$, respectively].

In Figs. 9(c) and 9(d), the evolution of spectral splitting in the spectral profile of the eleventh and the fifteenth harmonic order is shown for a focus position $z>0 \mathrm{~mm}$ at different pump-probe time delays, respectively. In this measurement, the intensity of the probe pulse was set to $7.0 \times 10^{14} \mathrm{~W} / \mathrm{cm}^{2}$. Similar to Figs. 9(a) and 9(b), at delays $\tau \leqslant 1.0$ ns no splitting effect is observed. With increasing time delay from $2.0 \mathrm{~ns}$ to $6.0 \mathrm{~ns}$, spectral splitting can be observed.

Due to the fact that spectral broadening, splitting, and blueshift are dependent on the pump-probe time delay, we attribute these three effects to transient phase matching (TPM) [47,49-52]. In TPM, the fundamental pulse causes a rapid ionization of the medium and thus a variation of the density of the free electrons. Additionally, this leads to temporally varying phase-matching conditions, where the HHG conversion efficiency maximizes at two instances over the pulse duration. As a result, the emitted radiation is spectrally split and blueshifted. These effects are observed when the creation of free electrons plays an important role. Thus, our measurement indicates that delay times up to $\tau=21.5 \mathrm{~ns}$ lead to a higher number of free electrons. There are two possible explanations. Avalanche ionization in the laser ignited plasma may take place. On the other hand, the calculated density of the target decreases by more than three orders of magnitude from $7.9 \times 10^{19} \mathrm{~cm}^{-3}$ at $\tau=1.5 \mathrm{~ns}$ to $4.0 \times 10^{16} \mathrm{~cm}^{-3}$ at $\tau=21.5 \mathrm{~ns}$ [Figs. 9(a) and 9(b)] and by more than two orders of magnitude from $2.2 \times$ $10^{20} \mathrm{~cm}^{-3}$ at $\tau=1.0 \mathrm{~ns}$ to $1.7 \times 10^{18} \mathrm{~cm}^{-3}$ at $\tau=6.0 \mathrm{~ns}$ [Figs. 9(c) and 9(d)]. The lower target density at long delay

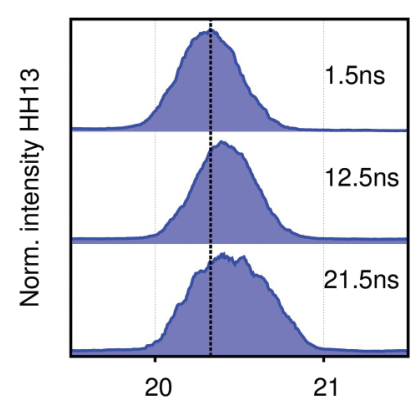

Photon energy (eV)

(a)

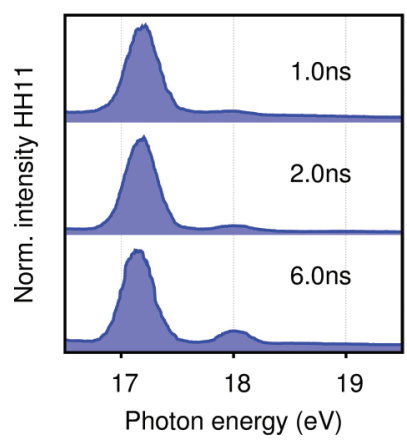

(c)

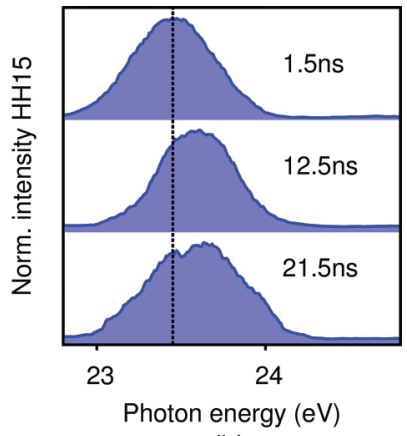

(b)

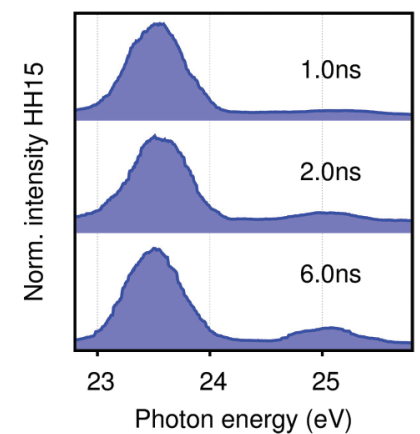

(d)
FIG. 9. (Color online) Blueshift and spectral broadening of the thirteenth harmonic order (a) and the fifteenth harmonic order (b) with respect to the pump-probe time delay. The dashed line indicates the nonshifted spectral line. The spectra were taken at an intensity of the probe pulse of $3.6 \times 10^{14} \mathrm{~W} / \mathrm{cm}^{2}$ at a focal position of $z<0 \mathrm{~mm}$. (c) and (d) Evolution of spectral splitting of the eleventh harmonic order (c) and of the fifteenth harmonic order (d) with respect to different pump-probe time delays. The spectra were taken at an intensity of the probe pulse of $7.0 \times 10^{14} \mathrm{~W} / \mathrm{cm}^{2}$ at a focal position of $z>0 \mathrm{~mm}$.

times may allow easier access of the probe pulse to the interior part of the droplet, which may otherwise be screened by the plasma at the droplet surface. This affects not only the generation of harmonic radiation but also increases the overall ionization induced by the probe pulse. Consequently, the density of free electrons rises with time. As a result, TPM conditions are achieved for the probe pulse and the effect becomes more pronounced for larger delays.

\section{CONCLUSION}

In this paper we have demonstrated phase-matching experiments on HHG from micrometer-sized liquid water droplets. In pump-probe experiments, we generate harmonic radiation up to the twenty-seventh harmonic order and find a strong dependency of the harmonic yield on the spatiotemporal behavior of the target, in agreement with Flettner et al. From calculations we estimate the density of the target as function of intensity and time. We detect harmonic radiation even at small delay times, where a high-density target is present and find a maximum in the harmonic yield around a pump-probe delay of $\tau=12.5 \mathrm{~ns}$. At a pump-probe time delay of $\tau=1.0 \mathrm{~ns}$, we study the influence of the focal position on efficient HHG and find a phase-matching behavior similar 
to gas jets [46], which agrees with our calculations of the target density. In intensity-dependent studies concerning the pump-pulse intensity, we identified two aspects influencing the harmonic yield: the intensity-dependent expansion of the droplet and the induced phase mismatch by free electrons. A variation of the probe-pulse intensity showed a saturation of the harmonic yield, which can be attributed to a rising density of free electrons, induced by the probe pulse. With increasing pump-probe time delay, spectral splitting, spectral broadening, and a blueshift of the harmonic radiation become observable, as already demonstrated in gas-phase experiments [47,49-52]. The dependence on the time delay indicates that these three effects can be attributed to transient phase matching.

The liquid droplet is a versatile target for HHG in a medium with variable thermodynamic state. Especially at short delays between pump and probe pulse, the configuration of the target will be of major interest for future experiments. Since we start with a liquid target and perform a transition into the gas phase, it is unknown what happens at short time delays. Due to its per- manent dipole momentum, the water molecule tends to cluster and a breakup of the droplets into large particles at short delays seems possible. Understanding these spatiotemporal dynamics within the droplet will be important for future experiments. The true benefits of the droplet are the mass limitation in combination with the debris-free HHG. As a light source for spectroscopy, the liquid-droplet source can be an excellent tool. The droplet source is not limited to water as a target for HHG. Many liquids can be used. The droplet can be doped with other particles [53], such as large biological molecules.

\section{ACKNOWLEDGMENTS}

The authors thank Thomas Binhammer for fruitful discussions. The authors thank Christian Spielmann for discussion of the characteristics of emission from water droplets. This work was partly funded by Deutsche Forschungsgemeinschaft (DFG) within the Cluster of Excellence QUEST, Centre for Quantum Engineering and Space-Time Research, and by DFG Project No. KO 3798/1-1.
[1] M. Ferray, A. L'Huillier, X. Li, L. Lompre, G. Mainfray, and C. Manus, J. Phys. B: At., Mol. Opt. Phys. 21, L31 (1988).

[2] P. B. Corkum, Phys. Rev. Lett. 71, 1994 (1993).

[3] M. Lewenstein, P. Balcou, M. Y. Ivanov, A. L'Huillier, and P. B. Corkum, Phys. Rev. A 49, 2117 (1994).

[4] M. Hentschel, R. Kienberger, C. Spielmann, G. A. Reider, N. Milosevic, T. Brabec, P. Corkum, U. Heinzmann, M. Drescher, and F. Krausz, Nature (London) 414, 509 (2001).

[5] R. Kienberger, E. Goulielmakis, M. Uiberacker, A. Baltuska, V. Yakovlev, F. Bammer, A. Scrinzi, T. Westerwalbesloh, U. Kleineberg, U. Heinzmann, M. Drescher, and F. Krausz, Nature (London) 427, 817 (2004).

[6] M. Schultze et al., Science 328, 1658 (2010).

[7] T. Ditmire, E. T. Gumbrell, R. A. Smith, J. W. G. Tisch, D. D. Meyerhofer, and M. H. R. Hutchinson, Phys. Rev. Lett. 77, 4756 (1996).

[8] L. Le Déroff, P. Salières, B. Carré, D. Joyeux, and D. Phalippou, Phys. Rev. A 61, 043802 (2000).

[9] M. Bellini, C. Lyngå, A. Tozzi, M. B. Gaarde, T. W. Hänsch, A. L'Huillier, and C.-G. Wahlström, Phys. Rev. Lett. 81, 297 (1998).

[10] C. Lyngå, M. B. Gaarde, C. Delfin, M. Bellini, T. W. Hänsch, A. L'Huillier, and C.-G. Wahlström, Phys. Rev. A 60, 4823 (1999).

[11] J.-F. Hergott, M. Kovacev, H. Merdji, C. Hubert, Y. Mairesse, E. Jean, P. Breger, P. Agostini, B. Carré, and P. Salières, Phys. Rev. A 66, 021801 (2002).

[12] E. Takahashi, Y. Nabekawa, and K. Midorikawa, Opt. Lett. 27, 1920 (2002).

[13] V. Tosa, E. Takahashi, Y. Nabekawa, and K. Midorikawa, Phys. Rev. A 67, 063817 (2003).

[14] H. J. Wörner, J. B. Bertrand, P. Hockett, P. B. Corkum, and D. M. Villeneuve, Phys. Rev. Lett. 104, 233904 (2010).

[15] T. Kanai, S. Minemoto, and H. Sakai, Nature (London) 435, 470 (2005).
[16] R. Torres, N. Kajumba, J. G. Underwood, J. S. Robinson, S. Baker, J. W. G. Tisch, R. de Nalda, W. A. Bryan, R. Velotta, C. Altucci, I. C. E. Turcu, and J. P. Marangos, Phys. Rev. Lett. 98, 203007 (2007).

[17] R. Velotta, N. Hay, M. B. Mason, M. Castillejo, and J. P. Marangos, Phys. Rev. Lett. 87, 183901 (2001).

[18] C. Vozzi, M. Negro, F. Calegari, G. Sansone, M. Nisoli, S. De Silvestri, and S. Stagira, Nature Phys. 7, 822 (2011).

[19] O. Smirnova, Y. Mairesse, S. Patchkovskii, N. Dudovich, D. Villeneuve, P. Corkum, and M. Ivanov, Nature (London) 460, 972 (2009).

[20] J. Itatani, J. Levesque, D. Zeidler, H. Niikura, H. Pepin, J. Kieffer, P. Corkum, and D. Villeneuve, Nature (London) 432, 867 (2004).

[21] S. Haessler, J. Caillat, W. Boutu, C. Giovanetti-Teixeira, T. Ruchon, T. Auguste, Z. Diveki, P. Breger, A. Maquet, B. Carre, R. Taieb, and P. Salieres, Nature Phys. 6, 200 (2010).

[22] A. Flettner, T. Pfeiffer, D. Walter, C. Winterfeld, C. Spielmann, and G. Gerber, Appl. Phys. B 77, 747 (2003).

[23] A. DiChiara, E. Sistrunk, T. Miller, P. Agostini, and L. DiMauro, Opt. Express 17, 20959 (2009).

[24] M. C. H. Wong, J.-P. Brichta, and V. R. Bhardwaj, Opt. Lett. 35, 1947 (2010).

[25] M. Falge, V. Engel, and M. Lein, Phys. Rev. A 81, 023412 (2010).

[26] S.-F. Zhao, C. Jin, R. R. Lucchese, A.-T. Le, and C. D. Lin, Phys. Rev. A 83, 033409 (2011).

[27] J. P. Farrell, S. Petretti, J. Förster, B. K. McFarland, L. S. Spector, Y. V. Vanne, P. Decleva, P. H. Bucksbaum, A. Saenz, and M. Gühr, Phys. Rev. Lett. 107, 083001 (2011).

[28] H. Sakai and K. Miyazaki, Appl. Phys. B 61, 493 (1995).

[29] Y. Akiyama, K. Midorikawa, Y. Matsunawa, Y. Nagata, M. Obara, H. Tashiro, and K. Toyoda, Phys. Rev. Lett. 69, 2176 (1992).

[30] T. D. Donnelly, T. Ditmire, K. Neuman, M. D. Perry, and R. W. Falcone, Phys. Rev. Lett. 76, 2472 (1996). 
[31] R. Ganeev, M. Suzuki, M. Baba, and H. Kuroda, Appl. Phys. Lett. 86, 131116 (2005).

[32] S. Ghimire, A. D. DiChiara, E. Sistrunk, P. Agostini, L. F. DiMauro, and D. A. Reis, Nature Phys. 7, 138 (2011).

[33] F. Quéré, C. Thaury, P. Monot, S. Dobosz, P. Martin, J.-P. Geindre, and P. Audebert, Phys. Rev. Lett. 96, 125004 (2006).

[34] P. Salières, A. L'Huillier, and M. Lewenstein, Phys. Rev. Lett. 74, 3776 (1995).

[35] E. J. Takahashi, T. Kanai, K. L. Ishikawa, Y. Nabekawa, and K. Midorikawa, Phys. Rev. Lett. 99, 053904 (2007).

[36] N. Papadogiannis, C. Kalpouzos, E. Goulielmakis, G. Nersisyan, D. Charalambidis, F. Auge, F. Weihe, and P. Balcou, Appl. Phys. B 73, 687 (2001).

[37] W. Boutu, T. Auguste, J. P. Caumes, H. Merdji, and B. Carré, Phys. Rev. A 84, 053819 (2011).

[38] V. V. Strelkov, V. T. Platonenko, and A. Becker, Phys. Rev. A 71, 053808 (2005).

[39] M. Berglund and L. Rymell, Rev. Sci. Instrum. 69, 1998 (1998).

[40] C. Weber, J. Appl. Math. Mech. 11, 136 (1931).

[41] J. L. Krause, K. J. Schafer, and K. C. Kulander, Phys. Rev. Lett. 68, 3535 (1992).

[42] H. Puell, Z. Naturforsch. A 25, 1807 (1970).
[43] S. Düsterer, Ph.D. thesis, Friedrich Schiller Universität Jena, 2002.

[44] C. Sarpe-Tudoran, A. Assion, M. Wollenhaupt, M. Winter, and T. Baumert, Appl. Phys. Lett. 88, 261109 (2006).

[45] C. Spielmann (private communication).

[46] P. Salières, P. Antoine, A. de Bohan, and M. Lewenstein, Phys. Rev. Lett. 81, 5544 (1998).

[47] C. Altucci, T. Starczewski, E. Mevel, C.-G. Wahlström, B. Carré, and A. L'Huillier, J. Opt. Soc. Am. B 13, 148 (1996).

[48] A. L'Huillier, M. Lewenstein, P. Salières, P. Balcou, M. Y. Ivanov, J. Larsson, and C. G. Wahlström, Phys. Rev. A 48, R3433 (1993).

[49] C. Altucci, R. Bruzzese, C. de Lisio, M. Nisoli, S. Stagira, S. De Silvestri, O. Svelto, A. Boscolo, P. Ceccherini, L. Poletto, G. Tondello, and P. Villoresi, Phys. Rev. A 61, 021801 (1999).

[50] Y. Wang, Y. Liu, X. Yang, and Z. Xu, Phys. Rev. A 62, 063806 (2000).

[51] F. Zhong, J. Deng, X. Hu, Z. Li, Z. Zhang, and Z. Xu, Phys. Lett. A 278, 35 (2000).

[52] D. S. Steingrube, T. Vockerodt, E. Schulz, U. Morgner, and M. Kovacčev, Phys. Rev. A 80, 043819 (2009).

[53] H. Chapman et al., Nature (London) 470, 73 (2011). 